\title{
Imaging X-ray Polarimetry Explorer (IXPE) Risk Management
}

\author{
Cheryl Alexander \\ Linc Research \\ NASA MSFC \\ Huntsville, AL 35812 USA \\ 256-961-7674 \\ cheryl.alexander@nasa.gov
}

\author{
Primo Attina \\ INAF-OATo \\ Pino Torinese (To), Italy \\ [39] (011) 810 -1952 \\ attina@oato.inaf.it
Ettore Del Monte
INAF-IAPS
Rome, Italy \\ [39] (064) 993-4675 \\ ettore.delmonte@iaps.inaf.it
}

\author{
Anthony Kelley \\ NASA MSFC \\ Redstone Arsenal \\ Huntsville, AL 35812 USA \\ (256) 544-7646 \\ anthony.kelley@nasa.gov
}

\author{
William D Deininger \\ Ball Aerospace \\ 1600 Commerce Street \\ Boulder, CO 80301 USA \\ 303-939-5314 \\ wdeining@ball.com
}

Mike Bowen

MTS, Inc.

NASA MSFC

Huntsville, AL 35812 USA

256-961-7799

mike.d.bowen@nasa.gov

\section{Lindsey Ingram \\ NASA MSFC}

Redstone Arsenal

Huntsville, AL, USA 35812

(256) 961-7023

lindsey.j.ingram@nasa.gov

\author{
Steve Pavelitz \\ NASA MSFC \\ Redstone Arsenal \\ Huntsville, AL 35812 USA \\ (256) 961-7462 \\ steven.d.pavelitz@nasa.gov
}

\author{
Randy Baggett \\ NASA MSFC \\ Redstone Arsenal \\ Huntsville, AL 35812 USA \\ 256-961-7404 \\ randy.baggett@nasa.gov
}

\author{
Chris Cowart \\ NASA MSFC \\ Redstone Arsenal \\ Huntsville, AL 35812 USA \\ 256-544-8901 \\ chris.cowart@nasa.gov \\ William Kalinowski \\ Ball Aerospace \\ 1600 Commerce Street \\ Boulder, CO, USA 80301 \\ 303-939-7161 \\ wkalinow@ball.com \\ Martin C. Weisskopf \\ NASA MSFC \\ Redstone Arsenal \\ Huntsville, AL 35812 USA \\ (256) 961-7798 \\ martin.c.weisskopf@nasa.gov
}

\begin{abstract}
The Imaging X-ray Polarimetry Explorer (IXPE) project is an international collaboration to build and fly a polarization sensitive $X$-ray observatory. The IXPE Observatory consists of the spacecraft and payload. The payload is composed of three $\mathrm{X}$-ray telescopes, each consisting of a mirror module optical assembly and a polarization-sensitive $\mathrm{X}$-ray detector assembly; a deployable boom maintains the focal length between the optical assemblies and the detectors. The goal of the IXPE Mission is to provide new information about the origins of cosmic X-rays and their interactions with matter and gravity as they travel through space. IXPE will do this by exploiting its unique capability to measure the polarization of $\mathrm{X}$-rays emitted by cosmic sources.
\end{abstract}

The collaboration for IXPE involves national and international partners during design, fabrication, assembly, integration, test, and operations. The full collaboration includes NASA Marshall Space Flight Center (MSFC), Ball Aerospace, the Italian Space Agency (ASI), the Italian Institute of Astrophysics and Space Planetology (IAPS)/Italian National Institute of Astrophysics (INAF), the Italian National Institute for Nuclear Physics (INFN), the University of Colorado (CU) Laboratory for Atmospheric and Space Physics (LASP), Stanford University, McGill University, and the Massachusetts Institute of Technology.
The goal of this paper is to discuss risk management as it applies to the IXPE project. The full IXPE Team participates in risk management providing both unique challenges and advantages for project risk management. Risk management is being employed in all phases of the IXPE Project, but is particularly important during planning and initial execution-the current phase of the IXPE Project. The discussion will address IXPE risk strategies and responsibilities, along with the IXPE management process which includes risk identification, risk assessment, risk response, and risk monitoring, control, and reporting.

\section{TABLE OF CONTENTS}

1. INTRODUCTION .......................................................2

2. BACKGROUND ..................................................2

3. IXPE RISK MANAGEMENT IMPLEMENTATION ....3

4. RISK MANAGEMENT PROCESS..............................5

5. CONCLUSION ........................................................ 10

ACKNOWLEDGEMENTS ......................................10

BIOGRAPHY .......................................................11

APPENDIX A IXPE RISK REGISTER INPUTS............. 14

APPENDIX B IXPE DRAFT RISK INPUTS................... 14 


\section{INTRODUCTION}

The goal of this paper is to describe risk management as it is being implemented for IXPE during the current NASA project life-cycle phase: Phase B, Preliminary Design and Technology Development. Following the introduction (Section 1), the paper begins with a background discussion of the IXPE mission: the international collaboration, science goals, project overview, and mission operations overview (Section 2) [1-9]. The background is immediately followed by a discussion of how risk management is implemented within IXPE, including the overall risk strategy approach and roles and responsibilities (Section 3). The bulk of the paper (Section 4) walks step-by-step through the IXPE risk process. The section begins with an overview that describes how risk management is accomplished given that IXPE is an international collaboration, with 3 major partners, one of which is an international contribution. The overview is followed by a walkthrough of the IXPE risk process steps: identification, assessment, response (a.k.a. handling strategy), monitoring, controlling, and reporting. Conclusions are discussed in Section 5.

There are two companion papers on IXPE at this conference. The first is a mission overview [9]; the second covers the systems engineering approach in use on IXPE [10].

\section{BACKGROUND}

The IXPE Mission is an international collaboration led by NASA MSFC as the Principal Investigator (PI) institution and includes Ball and CU/LASP, as well as the Italian Space Agency (ASI) with IAPS/INAF and INFN as major international partners who make up the IXPE Italian Team (I2T). The goal of IXPE is to expand understanding of highenergy astrophysical processes and sources, in support of NASA's first science objective in Astrophysics: "Discover How the Universe Works," by measuring the polarization of $\mathrm{X}$-rays emitted by cosmic sources. Polarization uniquely probes physical anisotropies-ordered magnetic fields, aspheric matter distributions, or general relativistic (GR) coupling to black-hole spin-that are not otherwise measurable.

\section{IXPE Project Overview [1-9]}

MSFC is providing the X-ray optics and Science Operations Center (SOC), along with mission management and systems engineering. Ball is responsible for payload Integration and Test (I\&T), the Spacecraft (S/C), system I\&T, launch, and operations. The Mission Operations Center (MOC) is located at CU/LASP. IAPS/INAF and INFN provide the unique polarization-sensitive gas pixel detectors (GPD) within the detector units (DU) and the Detectors Service Unit (DSU), which includes the payload computer.
The IXPE Observatory consists of the S/C and payload modules built up in parallel to form the Observatory during system integration and test.

IXPE's payload is a set of three identical X-ray telescopes mounted on a common optical bench and co-aligned with the pointing axis of the S/C. Each telescope operates independently, and is comprised of a 4-m-focal-length X-ray optic called a Mirror Module Assembly (MMA) that focuses $\mathrm{X}$-rays onto a polarization-sensitive GPD within its paired DU. A deployable boom maintains the focal length between the MMAs and DUs. Each DU contains its own electronics, which communicate with the DSU. The DSU bins the science data and interfaces with the S/C. Each DU has a multifunction filter wheel assembly for in-flight calibration checks and source flux attenuation.

The IXPE Observatory is based on the Ball BCP-100 S/C architecture, a low-cost, heritage approach with significant spacecraft capability and flexibility. The BCP-100 design supports the project goal of incorporating a low-risk spacecraft by using flight-proven components, a simple structural design, and significant design and software reuse from prior missions (STPSat-2, STPSat-3, and GPIM) [7-9]. The modular design allows for concurrent payload and S/C development with a well-defined, clean interface that reduces technical and schedule risk. IXPE is leveraging the flexibility of the BCP-100 architecture to accommodate the IXPE science payload. The IXPE payload is mounted on the S/C top deck. The IXPE Observatory is designed to launch on a Pegasus XL or larger launch vehicle.

Figure 1 shows the Observatory stowed in a Pegasus XL fairing and Figure 2 shows the IXPE Observatory on-orbit configuration.

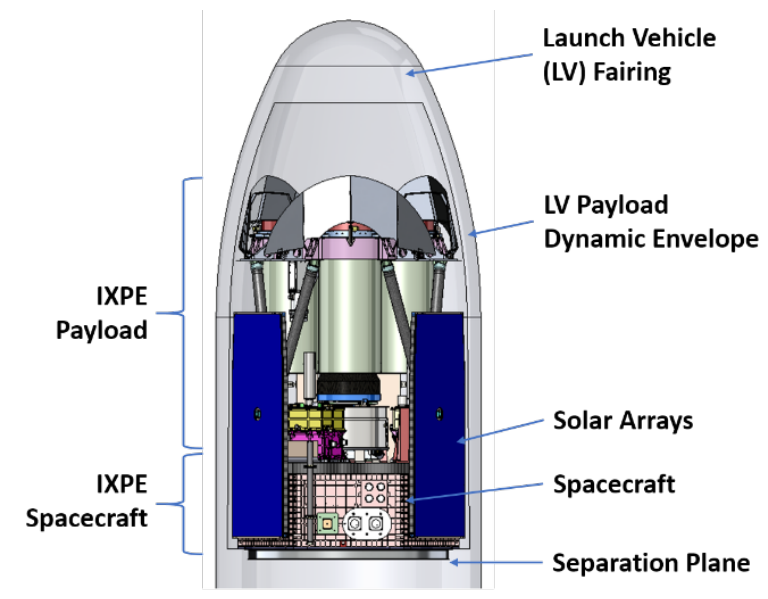

Figure 1. IXPE Observatory Stowed in Pegasus XL 


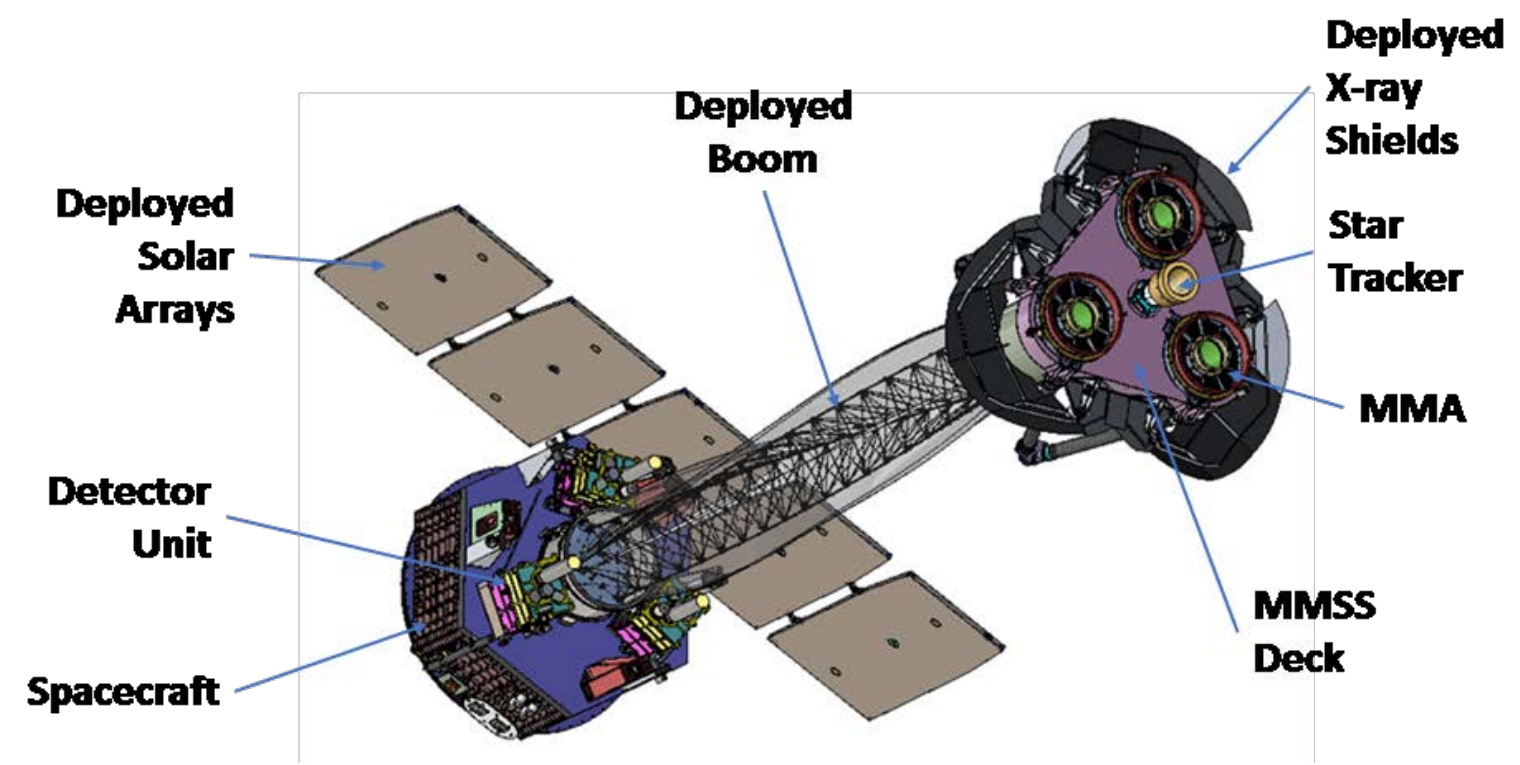

Figure 2. IXPE Observatory On-Orbit Configuration

\section{IXPE Mission Operations Overview}

IXPE is designed as a 2-year mission plus 30 days for commissioning with a current launch date of April 2021. IXPE launches to a circular low-Earth orbit at an altitude of $540 \mathrm{~km}$ and an inclination of 0 degrees. The payload uses a single science operational mode capturing the $\mathrm{X}$-ray data from the targets. The mission design follows a simple observing paradigm: pointed viewing of known X-ray sources (with known locations in the sky) over multiple orbits (not necessarily consecutive orbits) until the observation is complete. The Observatory communicates with the ASIcontributed Malindi ground station via S-band link. The science team generates and archives IXPE data products in NASA's High Energy Astrophysics Science Archive Research Center (HEASARC) using proven algorithms.

Additional information about IXPE can be found at: https://www.astro.msfc.nasa.gov/ixpe/.

\section{IXPE RISK MANAGEMENT IMPLEMENTATION}

The IXPE Project implements a risk management process that includes methodologies for identifying, analyzing, mitigating, monitoring, and tracking risks. As a cost-capped, Class D, fast-paced (Formulation to Launch in $\sim 4$ years) mission, these risk management methodologies are focused on providing project management the visibility needed to actively manage risks, and the insight required for robust, cost-based, risk-aware decision making.

The purpose of the risk management process is to minimize the probability and impact of adverse events which threaten project objectives. To be successful, risk management requires that all Project members actively engage in the process and ensure that risks are:
- Continuously identified throughout the Project life cycle

- Systematically analyzed using standardized criteria to determine impact and likelihood

- Appropriately prioritized to ensure the most effective use of Project resources

- Monitored and tracked to maintain an accurate Project risk profile and evaluate the effectiveness of the risk management process and risk-related activities.

These steps ensure the IXPE Project Manager (PM) can factor risk into day-to-day management of the Project and make effective cost-based, risk-aware decisions.

\section{Risk Strategy}

IXPE risk management practices are based on NASA's Continuous Risk Management (CRM) processes [11,12]. The IXPE project risk management process steps encompass risk identification, assessment (analyze), planning, tracking, and control per CRM (Figure 3) throughout the project life cycle.

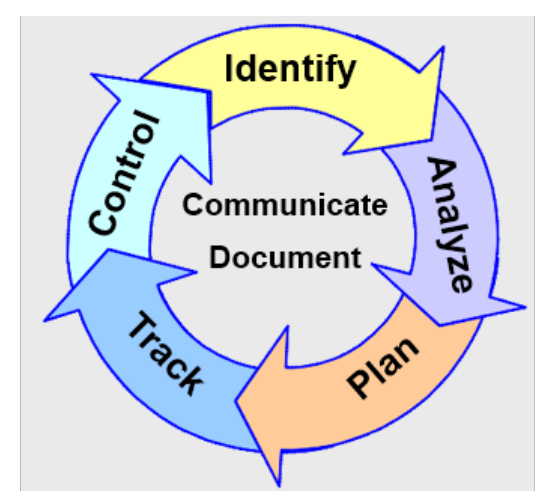

Figure 3. CRM Process 
Risks that potentially affect mission performance/technical margins or cost and schedule are tracked as threats to the budget, schedule, and/or mission/technical metrics until resolved, realized, or otherwise dispositioned.

The responsibilities and processes for managing risks associated with IXPE are documented in the IXPE Risk Management Plan (RMP), and is the subject of this paper. The IXPE RMP is implemented within the IXPE Project with full participation from the IXPE partners: MSFC, Ball Aerospace (Ball), and the I2T partners, and is applicable to risks associated with cost, schedule, and performance (technical/mission). Safety risks are incorporated into performance, cost, or schedule as appropriate.

\section{Roles and Responsibilities}

The IXPE PM serves as the Chair of the IXPE Risk Management Board (RMB), shown in Table 1, and is responsible for overall risk management and mitigation, and makes decisions with the concurrence of the Principal Investigator (PI). The PM screens all risks voted for inclusion in the risk register by the RMB, identifying risks requiring elevation for upper management visibility. Risks outside the scope of the project, or with significant cost, schedule, and/or technical impacts are presented to MSFC's Center Management and the Goddard Space Flight Center (GSFC) Explorer's Program Office (ExPO).

The PM delegates the leadership of technical risk identification, trade study oversight, and risk mitigation to the IXPE Project Systems Engineer (PSE), who also serves as the alternate RMB Chair. The PSE is responsible for ensuring technical risk mitigation strategies are appropriate and technical implementations are adequate.

The PM delegates the responsibility of the development and implementation of the risk management process to the IXPE Project Integration Lead, who serves as the project's Risk Lead (RL). The RL serves as the focal point for project team inputs and proposed mitigation strategies. The RL works with team members to develop risks, track open risks, maintain the project risk management tool (IXPE risk register), run monthly RMB meetings, track risk actions, and finalize RMB minutes. The RL develops and maintains the IXPE Risk Management Plan (RMP) for PM and RMB approval, and submits monthly reports to the PM for Project, Program, and MSFC reviews.

The PM delegates the responsibility of RMB Secretariat to the IXPE Configuration Management (CM) lead; the Secretariat takes the RMB meeting attendance, records minutes, and records actions.

The IXPE project team is responsible for identification and documentation of risks, assessment of identified risks for impacts to their respective areas, supporting the development of mitigation strategies for "Medium" and "High" risks, and reporting on risk mitigation status.
The IXPE RMB provides the forum for discussing project risks and makes decisions regarding the disposition of those risks. RMB members are the individuals established and authorized per the IXPE RMB Charter.

Table 1. IXPE Risk Management Board Membership

\begin{tabular}{|l|l|l|}
\hline Organization & \multicolumn{1}{|c|}{ Title } & Responsibility \\
\hline MSFC & Project Manager & Chair \\
\hline MSFC & $\begin{array}{l}\text { Project Systems } \\
\text { Engineer }\end{array}$ & $\begin{array}{l}\text { Alternate Chair, } \\
\text { Member }\end{array}$ \\
\hline MSFC & $\begin{array}{l}\text { Project Integration } \\
\text { Lead }\end{array}$ & Risk Lead \\
\hline MSFC & $\begin{array}{l}\text { Configuration } \\
\text { Management Lead }\end{array}$ & Secretariat \\
\hline MSFC & Principal Investigator & Member \\
\hline MSFC & $\begin{array}{l}\text { Lead Systems } \\
\text { Engineer }\end{array}$ & Member \\
\hline Ball & Ball Project Manager & Member \\
\hline Ball & $\begin{array}{l}\text { Ball Lead Systems } \\
\text { Engineer }\end{array}$ & Member \\
\hline IAPS/INFN & I2T Project Manager & Member \\
\hline IAPS/INAF & $\begin{array}{l}\text { I2T Lead Systems } \\
\text { Engineer }\end{array}$ & Member \\
\hline MSFC & Payload Manager & Member \\
\hline MSFC & $\begin{array}{l}\text { CSO/SMA } \\
\text { Representative }\end{array}$ & Member \\
\hline ASI & ASI SMA Lead & Member \\
\hline
\end{tabular}

Each RMB member is responsible for the following:

(1) Risk identification

(2) Regular review of risk status, scoring, and ranking

(3) Approval of specific risk handling strategies

(4) Evaluation of mitigation plans before and during plan execution

(5) Formulating recommendations regarding risk mitigation

(6) Review and approval of changes to the RMP

Risk decisions are made by consensus of the RMB. If consensus cannot be reached, the PM, in concurrence with the PI, has ultimate authority to make risk decisions and changes. If consensus cannot be reached between the PI and $\mathrm{PM}$, the risk issue is elevated to MSFC management unless the IXPE science return will be affected, in which case the PI has ultimate authority. 


\section{Risk MANAgement Process}

\section{Overview}

The IXPE risk management process involves full participation from all the IXPE partners: MSFC, Ball, and the I2C partners. Ball is the prime contractor for the IXPE mission. As such, IXPE risks involving Ball are assessed for cost, schedule, or performance impacts to the scope described in their contract; IXPE Project cost and schedule liens and/or contract modifications are implemented as required. Ball internal risk management processes are governed by the IXPE contract, derived from the Ball Quality Business System Risk and Opportunity Management Work Instruction, and described in the Ball Risk Management Plan for IXPE.

The I2T contribution involves contributions from multiple Italian organizations (ASI, IAPS/INAF, and INFN). The major I2T funding source and management authority is ASI.

As such, I2T works in a risk process governed and reported to ASI. The I2T risk processes are governed by the European
Cooperation for Space Standardization Risk Management, ASI ECSS-M-80C, and described in the IXPE Instrument Risk Management Plan. As a participant in the IXPE risk management process, I2T has adopted the IXPE risk ranking criteria, submits all risks reported to ASI to the IXPE RL, and participates fully in IXPE RMB activities. Since the partnership with I2T is an international contribution, IXPE risks involving I2T do not typically carry a cost "score" in the IXPE risk register (Appendix A) since cost mitigation liens carried by I2T/ASI do not affect Project reserves. All I2T risks are reviewed by the RMB to determine if it is appropriate for the Project to assign a cost score to a particular I2T risk due to potentially incurred costs by MSFC and/or Ball. If a risk involving I2T requires mitigation for which MSFC or Ball participation is required, risk "ownership" (i.e. the risk owner) is transferred from I2T to MSFC or Ball, as appropriate, and a cost score is assigned.

The IXPE risk management process is described in the following sections. An overview of the IXPE risk management process is shown in Figure 4.

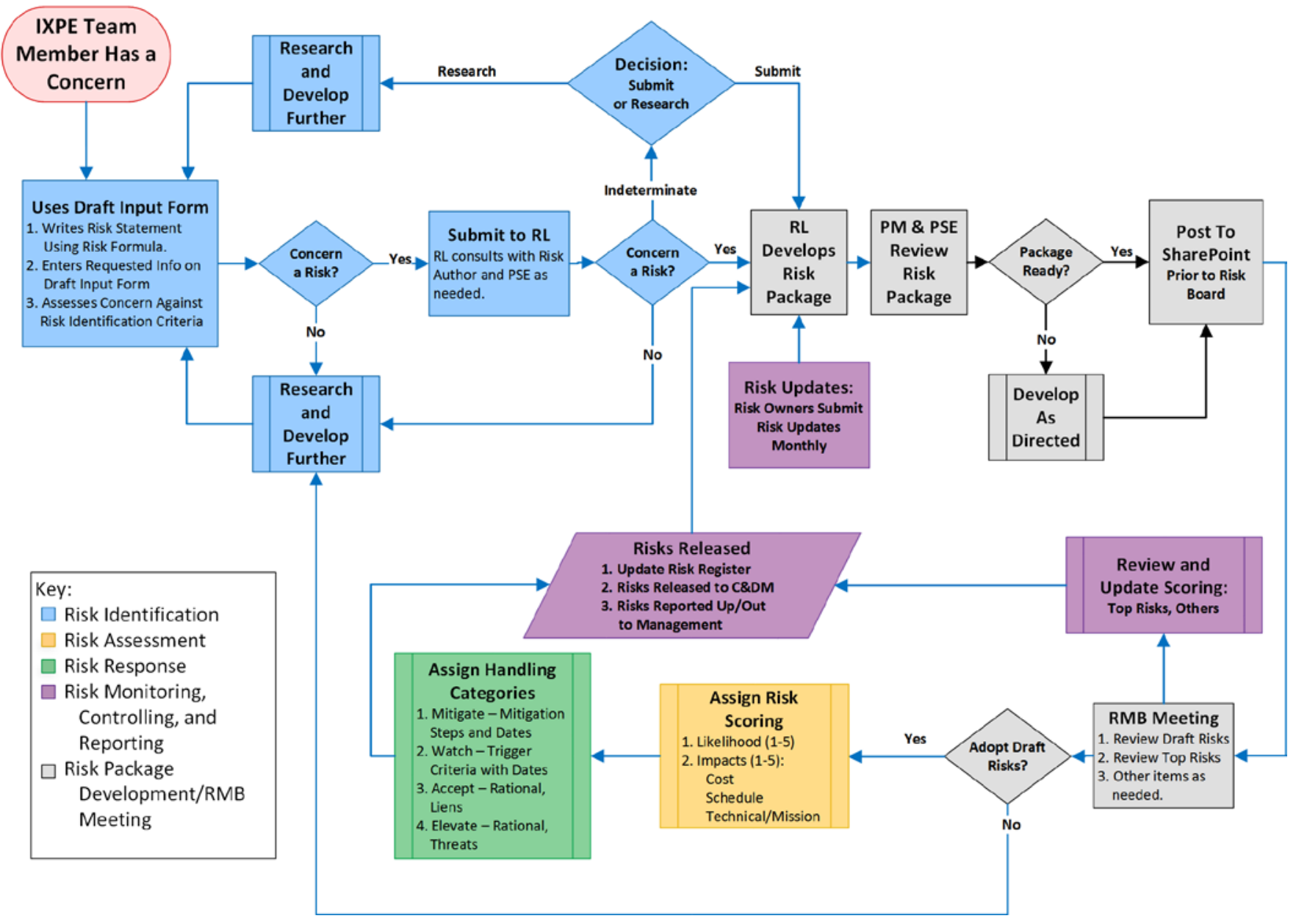

Figure 4. IXPE Risk Management Process 


\section{Risk Identification}

For the IXPE project, "risk" is defined as any scenario that, if/when encountered, may have a negative impact on the project's goals and objectives. Such scenarios may lead to degraded performance with respect to one or more performance measures (e.g., mission failure; inability to meet threshold mission requirements; exceeding mass or power limits, cost overruns, schedule slippage, etc.). Risk identification can occur at any point during the project life cycle and all IXPE team members can identify risks to be brought forward to the RMB. Activities for identifying risks on the IXPE Project include, but are not limited to, the following:

- Review of Documents

- Review of Designs

- Review of Change Requests

- Review of Test Data

- Formal Reviews

- Peer Reviews

- Technical Interchange Meetings

- Brainstorming

- Interviewing

- Assumption Analysis

- Review of Technical Requirements Documents

- Review of Payload Requirements Documents

- Review of Statements of Work

- Review of Spacecraft Level Specification

- Review of Spacecraft Component Product Functional Specifications, SOWs, and/or ICDs/IRDs

- Mission Assurance Plan

- Review of Project Security Plan

- Review of System Safety Plan

- Review of Environmental Design and Verification Requirements

- Review of Interface Control Documents

- Review of Interface Requirements Documents

- Review of Test Plans, Procedures, and Reports

- Review of Lessons-learned

Risk inputs are actively solicited prior to board meetings; RMB members are responsible for actively soliciting and collecting risks from the IXPE project teams they represent. Identified risks are submitted to the RL via a definitive risk statement (Table 2) accompanied by supporting information using a draft input form (Appendix A) and the process described below.

Risk Statement-For all identified risk scenarios (also referred to as "concerns" in this paper), the risk author writes a risk statement using a simple risk statement formula: "Given [CONDITION], there is a possibility that [CONSEQUENCE].” The risk statement captures the conditions that, if they exist, have the possibility of producing a particular undesired consequence (Table 2).
Table 2. IXPE Risk Statement Formula

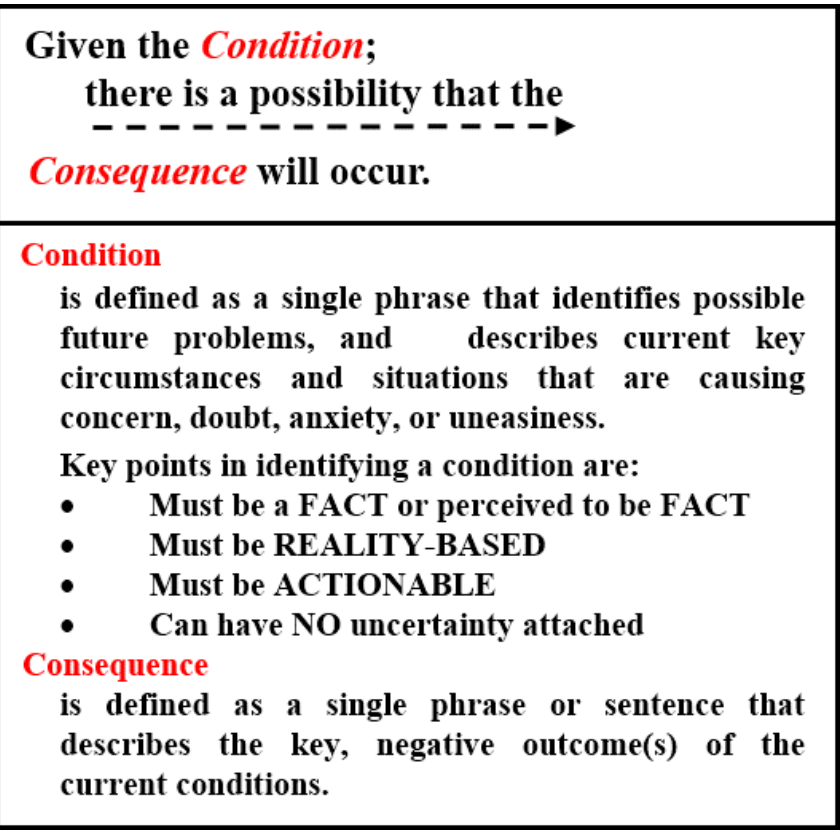

If the risk statement does not provide enough clarity, one of two longer formats is used [13]:

1) "Given that [CONDITION], there is a possibility of [DEPARTURE] adversely impacting [CONSEQUENCE]”

or

2) "Given that [CONDITION], there is a possibility of [DEPARTURE] adversely impacting [ASSET], thereby leading to [CONSEQUENCE]" where DEPARTURE describes a possible change from the baseline project plan, and ASSET is a primary resource that is affected.

Risk Identification Criteria-The IXPE team member is responsible for assessing his or her concern against the risk identification criteria (the six questions below). If the team member considers the concern to be a risk, the team member begins development of the risk by submitting a draft risk to the IXPE RL using a draft risk input form (Appendix B) available on the Project's SharePoint site. This is done approximately 1 week before the RMB meets or as determined by the RL with concurrence of the PM. The RL reviews the draft risks and may collaborate with the author of the draft risk and the PSE to jointly determine if it fully meets the criteria of a risk. If it is agreed that the draft risk meets the risk identification criteria, the RL works with the risk author to develop the draft risk for submission to the RMB. Draft risks which do not meet the risk identification criteria may be researched and resubmitted at a later date. If agreement cannot be reached on whether a draft risk meets the risk identification criteria, it may be presented to the RMB for discussion by the full board where, if it is not adopted as a risk, may also be assigned to be researched. This process ensures that Team members concerns are fully understood and assessed. 


\section{Risk Identification Questions/Criteria}

(1) Is the concern a risk? (Could it result in an undesirable situation or circumstance and have a realistic likelihood of occurring?)

(2) Is the concern already covered in forward work? (If so, it may not be a risk.)

(3) Can the concern be addressed by funds without going through the risk management process? (Management may decide to apply those funds and include it in forward work; therefore, may not be a risk, assuming the funded task provides full mitigation.)

(4) Is the concern a known risk (duplicative)? (Review the existing risk and, if necessary, update the description and context.)

(5) Is the concern an issue/problem? (Not a risk because the likelihood is $100 \%$ - the threat has already occurred.) IXPE tracks issues internally and reports issues to MSFC upper management (Science and Technology Office and Center Management Council), and the Explorers Program Office at GSFC.

(6) Is the concern a budget line or unfunded cost impact? (If it is, then it should be communicated to the PM and Business Manager.) Note: Unfunded cost impacts are also documented and tracked.

Additionally, all draft risks, even if they have been determined to meet the risk identification criteria by the RL and PSE, are discussed by the full board for assessment before being adopted as a risk. The team member who proposed the draft risk is responsible for presenting valid reasons for why it is a risk and not forward work, a duplicate risk, a problem, etc.

\section{Risk Assessment}

Risk Scoring Criteria-All IXPE draft risks, and risks subject to reassessment and ranking, are assigned a probability of occurrence, or likelihood, with associated ranked consequences for cost, schedule, and technical/mission (performance) impacts using the risk ranking criteria in Table 2 (likelihood/impacts/consequences) and Table 3 (mass and power margins). Safety risks are incorporated into cost, schedule, or performance, as appropriate, since IXPE is a non-human rated mission with no significant risks to humans or personal safety requirements. The criteria were developed specifically for IXPE and approved by the RMB for use during the current phase of development (NASA Life-cycle Phase B: Preliminary Design and Technology Development). At the time when the Project passes from one phase to another (Phase B to C, C to D, etc.) the "risk ranking criteria" will be reassessed and impact/consequence rankings will be adjusted proportional to the Project's budget, schedule, and mass/power reserve for that phase.

Risk Scoring-Using Tables 3 \& 4, IXPE risks are scored in accordance with the following:

(1) Determine likelihood scores (1-5) and impact/consequence scores (1-5) using IXPE's risk ranking criteria (Tables $3 \& 4$ ).

(2) Calculate the "composite risk score" by multiplying the likelihood by the highest impact/consequence score (composite scores range from a minimum of 1 to a maximum of 25).

(3) Composite scores are mapped to a 5x5 matrix (Figure 5) where, for reporting purposes: Green = "Low" risks, Yellow = "Medium" risks, and Red = "High" risks.

(4) Risks that have a composite score of 9 or above are considered significant by the IXPE RMB (encompasses all red risks and some yellow risks). These significant risks are reported out and up on a monthly basis as "top risks" to management at MSFC, and the GSFC ExPO.

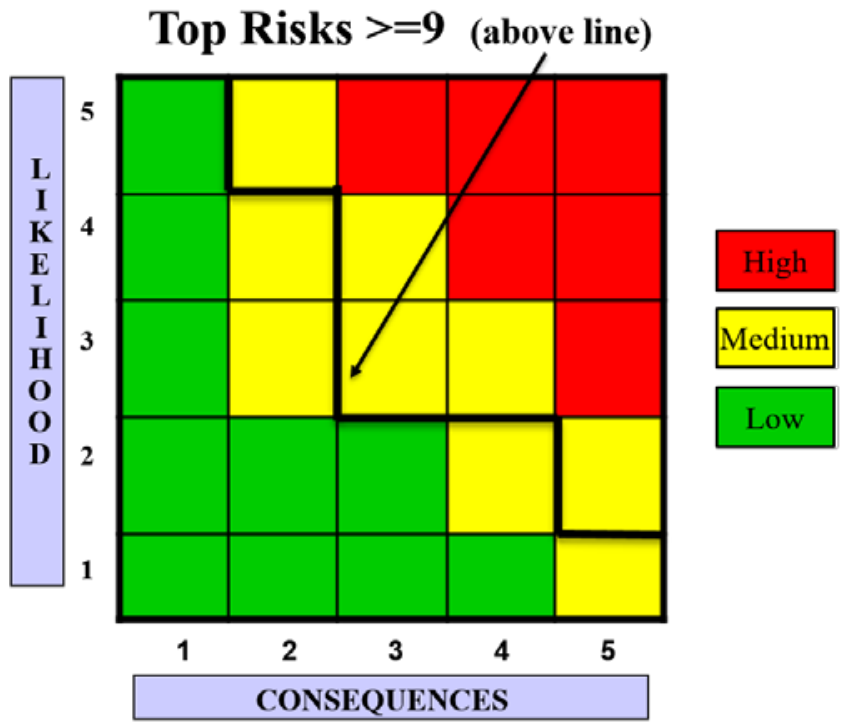

Figure 5. IXPE 5x5 Matrix; Top Risks 
Table 3. Risk Impact/Consequence Criteria

\begin{tabular}{|c|c|c|c|c|c|}
\hline \multirow[b]{2}{*}{$\begin{array}{l}\text { Risk } \\
\text { Level }\end{array}$} & \multirow[b]{2}{*}{ Likelihood } & \multicolumn{4}{|c|}{ Impacts } \\
\hline & & $\begin{array}{c}\text { Cost } \\
\text { Impacts }\end{array}$ & $\begin{array}{l}\text { Schedule } \\
\text { Impacts } \\
\text { (days late) }\end{array}$ & $\begin{array}{c}\text { Technical or } \\
\text { Mission Impacts }\end{array}$ & $\begin{array}{c}\text { Impacts } \\
\text { on Other } \\
\text { Teams }\end{array}$ \\
\hline 5 & $\begin{array}{l}\quad>90 \% \\
\text { Very High }\end{array}$ & $>\$ 3 M$ & $\begin{array}{l}\text { Inability to meet } \\
\text { major program } \\
\text { milestone } \\
\text { (>6 weeks) }\end{array}$ & $\begin{array}{l}\text { Mission failure or unacceptable experiment } \\
\text { performance (fails to meet threshold mission } \\
\text { requirements); threatens ability to meet minimum } \\
\text { mission success criteria; estimates exceed } \\
\text { established margins (mass, power, volume) }\end{array}$ & \\
\hline 4 & $\begin{array}{l}\text { 60-90\% } \\
\text { High }\end{array}$ & $\begin{array}{l}\$ 1 \mathrm{M} \text { to } \\
\$ 3 \mathrm{M}\end{array}$ & $\begin{array}{l}\text { Major slip, } \\
\text { affecting key } \\
\text { milestones / } \\
\text { critical path } \\
\text { (4-6 weeks) }\end{array}$ & $\begin{array}{l}\text { Inability to meet functional requirements of key } \\
\text { experiments; loss of science (major impact to full } \\
\text { success criteria, minimum success criteria is } \\
\text { achievable); threatens established margins. } \\
\text { Meets threshold mission with no margin. }\end{array}$ & \\
\hline 3 & 40-60\% & $\begin{array}{c}\$ 500 \mathrm{~K} \\
\text { to } \\
<\$ 1 \mathrm{M}\end{array}$ & $\begin{array}{l}\text { Minor slip } \\
\text { affecting key } \\
\text { milestones } \\
\text { (2-4 weeks) }\end{array}$ & $\begin{array}{l}\text { Degraded system performance; degraded mission } \\
\text { (does not meet baseline mission, meets threshold } \\
\text { mission requirements with margin); can handle } \\
\text { within established margins. }\end{array}$ & $\mathrm{Y} / \mathrm{N}$ \\
\hline 2 & $10-40 \%$ & $\begin{array}{c}\$ 200 \mathrm{~K} \\
\text { to } \\
<\$ 500 \mathrm{~K}\end{array}$ & $\begin{array}{l}\text { Slip affecting } \\
\text { minor milestones; } \\
\text { still able to meet } \\
\text { key need dates } \\
\text { (1-2 weeks) }\end{array}$ & $\begin{array}{l}\text { Ability to meet experiment requirements } \\
\text { affected; performance degraded; reduces margin } \\
\text { to full success criteria; can handle within } \\
\text { established margins. Meets baseline mission with } \\
\text { reduced margins. }\end{array}$ & \\
\hline 1 & $\begin{array}{c}<10 \% \\
\text { Very Low }\end{array}$ & $<\$ 200 \mathrm{~K}$ & $\begin{array}{l}\text { Small slip; no or } \\
\text { low impact to key } \\
\text { milestones } \\
\text { (<1 week) }\end{array}$ & $\begin{array}{l}\text { Minor effect or no impact; loss of non-critical } \\
\text { function; no impact on margins. }\end{array}$ & \\
\hline
\end{tabular}

Table 4. Additional Risk Impact/Consequence Criteria: Risk Mass and Power Margins

\begin{tabular}{|c|c|c|c|}
\hline Consequence & $\begin{array}{c}\text { Consequence } \\
\text { Level } \\
\text { Definition }\end{array}$ & $\begin{array}{c}\text { Observatory } \\
\text { Dry Mass }\end{array}$ & $\begin{array}{c}\text { Observatory } \\
\text { Power }\end{array}$ \\
\hline 5 & Very High & $>10 \mathrm{~kg}$ & $>20 \mathrm{~W}$ \\
\hline 4 & High & $>5 \mathrm{~kg}$ & $>10 \mathrm{~W}$ \\
\hline 3 & Moderate & $>2 \mathrm{~kg}$ & $>5 \mathrm{~W}$ \\
\hline 2 & Low & $>1 \mathrm{~kg}$ & $>1 \mathrm{~W}$ \\
\hline 1 & Very Low & $<1 \mathrm{~kg}$ & $<1 \mathrm{~W}$ \\
\hline
\end{tabular}




\section{Risk Response}

Once risks are identified and scored, they must be assigned a risk response, a.k.a. as a handling strategy. The risk author is responsible for proposing a response approach according to Table 5 and developing a response plan with actionable steps, a timeframe for implementation, possible trigger criteria, and planned closure date. The risk author presents this information to the RMB during the monthly meeting; the $\mathrm{RMB}$ collaborates and votes on whether the risk is included in the risk register and determines the risk scoring and what risk response will be implemented. If the risk is included in the register, a risk owner is assigned (often the risk author), and as such is responsible for overseeing the implementation of the agreed disposition of the risk.

Table 5. Response Approach Definitions

\begin{tabular}{|l|l|}
\hline Approach & \multicolumn{1}{|c|}{ Definition } \\
\hline $\mathrm{R}$ - Research & $\begin{array}{l}\text { Investigate the risk until enough is known } \\
\text { to decide what approach to take via } \\
\text { RIDM (i.e., mitigate, watch, accept). }\end{array}$ \\
\hline $\mathrm{M}-$ Mitigate & $\begin{array}{l}\text { Monitor the risks and their attributes for } \\
\text { early warning of critical changes in } \\
\text { impact, probability, timeframe, or other } \\
\text { aspects. }\end{array}$ \\
\hline $\mathrm{E}-$ Elevate & $\begin{array}{l}\text { Eliminate or reduce the risk by reducing } \\
\text { the impact, probability, or shifting the } \\
\text { timeframe. }\end{array}$ \\
\hline A - Accept & $\begin{array}{l}\text { Transfer the management of a risk to the } \\
\text { organizational level. } \\
\text { problem if it occurs. No further resources } \\
\text { are expended managing the risk. }\end{array}$ \\
\hline C - Close & $\begin{array}{l}\text { The risk no longer exists, it is no longer } \\
\text { cost effective to track as a risk, the risk is } \\
\text { superseded or encompassed by other } \\
\text { risks, or it has been mitigated. }\end{array}$ \\
\hline
\end{tabular}

For risks dispositioned as "Research," the RMB gives direction on what information is to be gathered or assessed to support an additional disposition and the timeframe in which it should occur. The risk owner is responsible for gathering the information unless otherwise directed and reporting back to the RMB according to the agreed upon timeline.

For risks dispositioned as "Watch," the RMB-or if so assigned, the RL, in collaboration with the PSE-identifies triggers (events or timeframes) that will provide early warning of significant changes in the likelihood or consequence of the risk. The risk is then reassessed according to the watch/trigger plan.

For risks dispositioned as "Mitigate," the risk author identifies ways to prevent the risk from occurring or reduce its impact or probability of occurring. Risk mitigations include prototyping, design modifications, additional verification and validation, adding tasks to the project schedule, release of margins, the development of a descope plan, etc. Mitigation steps must be actionable and have defined due dates. A mitigation actionee(s) will be identified and assigned by the board during the RMB meeting, usually the risk author/owner. Risk mitigation activities will be reportable to, and ultimately enacted and directed by, the PM. Approved mitigation plans that include use of schedule margin are approved by the PM and reviewed at monthly schedule meetings; use of schedule reserves is tracked in the Integrated Master Schedule. Mitigation plans that include the allocation of cost reserves are tracked by the Business Manager, approved by the PM, and presented during the IXPE Business Management Review. Mitigations that require the release of technical margins are approved by the Project Systems Engineering Team and reported in the IXPE Monthly Management Review. Formal, final release of all margins is approved by the IXPE Program Control Board.

For risks dispositioned as “Elevate,” the PM notifies MSFC management and/or the GSFC ExPO, as required; a risk may be dispositioned as "Elevate" if project resources or authority are insufficient for mitigation.

Risks designated as “Accept” are tracked by the project; liens for cost, schedule, and technical are levied as discussed below and reported up and out to MSFC management and ExPO. Risk accountability is assigned to either the PI or PM per NID 8001-108. For accepted risks related to mission success or safety, the decision is recorded in the risk register and clearly documented on a form in the IXPE CM system. The form contains at least the signature of the Risk Owner referencing the following: the case relied upon to justify the decision, and the assumptions, constraints, evaluation of aggregate risk, and the acceptance criteria on which the decision is based. The form includes the signatures of Technical Authorities (TAs) with their positions. If TAs disagree with the risk acceptance, they elevate the risk acceptance decision up the organizational hierarchy in accordance with the dissenting opinion process (NPD 1000.0).

Risks recommended for "Closure” must be accompanied by credible rationale to support closure. If the mitigation actions have eliminated the risk, the risk is superseded or encompassed by other risks, or the risk is no long considered to be a valid risk, it can be considered for closure. The PM is the final authority on the closure of all project risks.

For risks dispositioned as "Mitigate" which have been mitigated and the likelihood and consequence have been reduced to very low levels, the "residual" risk may be considered for "Acceptance.” If the risk is “Accepted” it will follow the protocol for Accepted risks discussed above.

For risks dispositioned as "Research" or "Watch,” a weighted cost exposure (i.e. the cost exposure scaled by the midrange probability of the assigned likelihood) is assessed as a threat against project reserves. For risks dispositioned as "Mitigate” which have fully defined mitigation costs accepted by the RMB and PM, the mitigation costs are assessed as a lien against project reserves. For risks dispositioned as “Mitigate” 
which have mitigations that include defining/refining the mitigation costs based on trade studies between mitigation strategies, vendors, etc., the initial weighted cost exposure is tracked as a threat against project reserves; when the mitigation costs are defined and accepted by the RMB and PM, the cost is tracked as a lien. For Risks dispositioned as "Accept" or "Elevate," the cost exposure/weighted cost exposure is tracked as a threat or lien as determined by the RMB and PM. All cost threats and liens are recorded in the risk register, tracked by the Business manager, and reported upward and out to STO and ExPO.

\section{Risk Monitoring, Controlling, and Reporting}

A key aspect of project risk control is accomplished through the formal monthly RMB reviews, during which relevant data is assessed to determine the effectiveness of mitigation plans and any needed adjustments. When needed, interim RMB meetings are called to deal with time-sensitive issues. Each risk owner is responsible for submitting ranking updates and risk status to the RL prior to RMB reviews. The RMB determines the criteria for top risks (currently a composite risk score of 9 or above). Top risks are assessed at a higher priority, reviewed at each monthly RMB meeting, reported up and out to MSFC management and ExPO, and have risk response planning, which may include both a risk mitigation and risk contingency plan. Contingency plans, once approved and initiated, are added to the project work plan and tracked and reported along with all other project activities. In addition to top risks, submitted draft risks and other risks, as determined by the RL or PM, are reviewed at monthly RMB meetings; the RL reviews all risks monthly to determine if there is reason for RMB review due to changing conditions or assumptions. The RMB reviews all project risks prior to major life cycle reviews (e.g., System Readiness Review, Preliminary Design Review, Critical Design Review, Operational Readiness Review, and Pre-Ship Review).

The current status and trending of top risks is presented at all project reviews, including the IXPE Monthly Management Review (MMR), the Science and Technology Office Monthly Project Review, MSFC Center Management Council Review, and the ExPO Status Review. Cost threats and liens for individual risks and the total project are presented at the MMR and to the ExPO.

\section{CONCLUSION}

Currently—Risk management for the IXPE Project Phase B Life-cycle has been described (CRM). Risk roles and responsibilities are defined and well understood by a team with risk management heritage (both the Ball LSE and I2C Payload Manager were former Risk Managers of space payload projects). Appropriate risk processes are in place (identification, assessment, response, monitoring, controlling, and reporting) and the team is fully engaged.

The IXPE Project is implementing a risk management process that includes methodologies for identifying, analyzing, mitigating, monitoring, and tracking risks. These risk management methodologies are focused on providing Project management the visibility needed to be risk aware and actively manage risks in a cost-capped, schedule driven environment.

Future-IXPE's Ball Partner will use Earned Value Management (EVM) beginning with Phase C. The current cost-based risk assessment process used by IXPE fits well with EVM to accurately estimate the cost of remaining work. Combined with actual costs for work performed to date, the estimate to complete will give the project an overall cost Estimate at Completion (EAC) that will fund projections / needs, staffing profiles, etc. The identified risks help quantify future impacts not yet captured in performance. As risk threats are identified, evaluated, statused, and ultimately mitigated when applicable, the EAC will be updated to reflect the projects latest revised estimate. Typically, this occurs on a monthly basis as part of the business rhythm and in accordance with the risk register updates.

\section{ACKNOWLEDGEMENTS}

The risk management process described here results from the combined efforts of teams at NASA MSFC, Ball Aerospace, ASI, INFN, and IAPS/INAF. The authors wish to thank NASA for funding the IXPE mission and to ASI for their significant contribution.

\section{REFERENCES}

[1] M. C. Weisskopf, Brian Ramsey, Stephen O’Dell, Allyn Tennant, Ronald Elsner, Paolo Soffita, Ronaldo Bellazzini, Enrico Costa, Jeffery Kolodziejczak, Victoria Kaspi, Fabio Muleri, Herman Marshall, Giorgio Matt, Roger Romani, "The Imaging X-ray Polarimetry Explorer (IXPE)," Proc. SPIE Vol. 9905, Space Telescopes and Instrumentation 2016: Ultraviolet to Gamma Ray, 990517 (11 July 2016).

[2] M. C. Weisskopf, Brian Ramsey, Stephen O’Dell, Allyn Tennant, Ronald Elsner, Paolo Soffita, Ronaldo Bellazzini, Enrico Costa, Jeffery Kolodziejczak, Victoria Kaspi, Fabio Muleri, Herman Marshall, Giorgio Matt, Roger Romani, "The Imaging X-ray Polarimetry Explorer (IXPE),” Results in Physics, Elsevier, Vol. 6, 31 Oct 2016, pp. 1179-1180.

[3] Paolo Soffitta, "IXPE the Imaging X-ray Polarimetry Explorer," Published in SPIE Proceedings Volume 10397: UV, X-Ray, and Gamma-Ray Space Instrumentation for Astronomy XX, September 2017.

[4] M. C. Weisskopf, for IXPE Team, "The Imaging X-ray Polarimetry Explorer (IXPE) - An Overview,” NASA SMD, Astrophysics Subcommittee, October 23, 2015.

[5] Brian Ramsey, M.C Weisskopf, R. Bellazzini, E. Costa, R. Elsner, V. Kaspi, H. Marshall, G. Matt, F. Muleri, G. Pavlov, S. O’Dell, G. Pavlov, R. Romani, P. Soffita, A. Tennant, N. Bucciantini, E. Churazov, M. Dovciak, R. 
Goosman, V. Karas, D. Lai, F. Marin, P-O. Petrucci, J. Poutanen, M. Salvati, L. Stella, R. Sunyaev, R. Turolla, $\mathrm{K}$. $\mathrm{Wu}$ and $\mathrm{S}$. Zane, "IXPE: The Imaging X-ray Polarimetry Explorer Implementing a Dedicated Polarimetry Mission,” Science Research Office (SRO), 2014.

[6] M. C. Weisskopf, R. Bellazzini, E. Costa, Brian Ramsey, S. O’Dell, A. Tennant, R. Elsner, G. Pavlov, G. Matt, H. Marshall, V. Kaspi, R. Romani, P. Soffita, F. Muleri, "IXPE: The Imaging X---ray Polarimeter Explorer Expanding Our View of the Universe,” SRO, AAS, 2014 HEAD, 116-15

[7] W. D. Deininger, R. Dissly, J. Domber, J. Bladt, J. Jonaitis, A. Kelley, R Baggett, B. D. Ramsey, S. L. O’Dell, M. C. Weisskopf and P. Soffitta, "Small Satellite Platform Imaging X-Ray Polarimetry Explorer (IXPE) Mission Concept and Implementation," SSC17-III-08, 31st Annual AIAA/USU Conference on Small Satellites - Small Satellites - Big Data, Logan, UT, USA, August 2017.

[8] William D Deininger, William Kalinowski, Zach Allen, Jeff Bladt, Mary Boysen, Kyle Bygott, Jennifer Erickson, John Ferguson, Larry Guy, Sandra Johnson, Huong Phan, Brian Smith, Jeffrey Wedmore, Janice Houston, Anthony Kelley and Brian Ramsey, "Imaging X-Ray Polarimetry Explorer Mission: Spacecraft Implementation Concept,” AIAA-2017-5314, 2017 AIAA SPACE and Astronautics Forum and Exposition, SATS-04, Small Satellites IV, September 2017.

[9] William D Deininger, William Kalinowski, Colin Peterson, Jeff Bladt, Brian Smith, Kyle Bygott, Larry Guy, Sandra Johnson, Zach Allen, Scott Mitchell, Darren Osborne, Allyn Tennant, Brian Ramsey, Janice Houston, Ettore Del Monte, Alessio Trois, “Observatory Design for the Imaging X-Ray Polarimeter Explorer (IXPE) Mission,” Paper 2.02.xx, 2018 IEEE Aerospace Conference, Big Sky MT, USA, 4-10 March 2018

[10] Janice Houston, William D Deininger, Jennifer Erickson, William Kalinowski, Ettore Del Monte, “Imaging X-ray Polarimetry Explorer Mission Overview and Systems Engineering Status,” Paper 2.12.xx, 2018 IEEE Aerospace Conference, Big Sky MT, USA, 4-10 March 2018

[11] NASA Interim Directive (NID) 8000-108, Agency Risk Management Procedural Requirements, NASA, 2016.

[12] NASA/SP-2011-3422, NASA Risk Management Handbook, NASA, 2011.

\section{BIOGRAPHY}

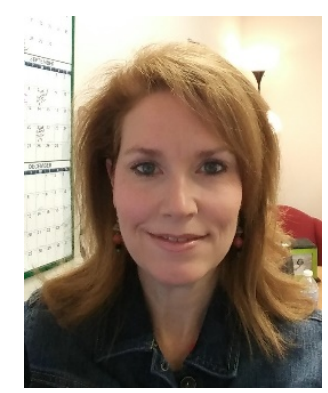

Cheryl Alexander received a B.S. in Engineering from the University of Alabama in Huntsville, in 1992; a Masters in Electrical Engineering in 1993, and has completed studies for a Ph.D. in Optical Science and Engineering. She began her career at NASA in 1990 as a co-op and later a full-time civil servant where she worked for 15 years on a variety of projects (Chandra, HERO, GOES Solar X-ray Imager, and SEDS) mostly centered around calibration and testing of $X$ ray optical systems and small optical systems design. Ms. Alexander left NASA and the space arena to pursue other interests in late 2005. In 2014 she joined Linc Research and began working again in optical systems and space missions. She joined the IXPE team during the Phase A Concept Study and successfully led the IXPE Cost Proposal. Currently, she is the IXPE Project Integration Lead and serves as the Risk Lead for IXPE Risk Management.

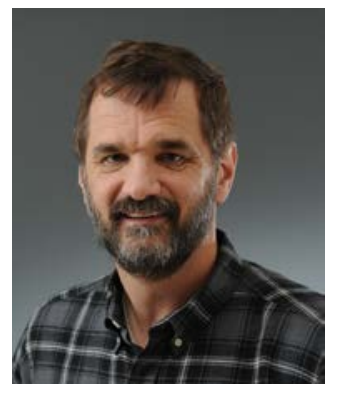

Bill Deininger is a staff consultant in systems engineering for Ball Aerospace. Dr. Deininger currently is the Ball Chief Systems Engineer (CSE) and Payload Lead on the NASA IXPE Project and a member of the IXPE Risk Management Board. He is also the Chief Engineer on the Green Propellant Infusion Mission. Recent work has included serving as the Ball CSE on the NEOCam Phase A Study, PI and systems lead for the Solar Electric Propulsion Technology Demonstration Mission study and systems lead for the CPST Mission study, all 3 for NASA. He was the functional manager for the Mission Systems Engineering group at Ball Aerospace for 2 years and, prior to that, the Kepler Flight Segment Manager. He was also the Chief Systems Engineer on the Mars Micromissions and StarLight Projects. Prior to joining Ball Aerospace, Dr. Deininger worked at FiatAvio-BPD in Italy for 9 years, as a Member of the Technical Staff at JPL for 8 years, and at Argonne National Laboratory for 11/2 years. He is an Associate Fellow of AIAA, Senior Member of IEEE and was awarded 2017 Engineer of the Year by the Rocky Mountain Section of the AIAA. He received a Dottorato di Ricerca (Ph.D.) in Aerospace Engineering from the Università degli Studi di Pisa in Italy, an M.S. in Plasma Physics from Colorado State University, and a B.S. in Physics from the State University of New York at Cortland. 


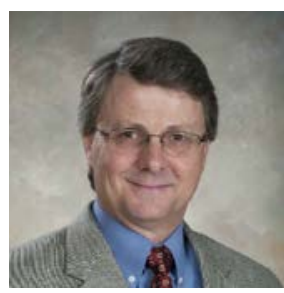

Randy Baggett is the Project Manager for IXPE and the chairman of the IXPE Risk Management Board. Mr. Baggett has been involved in space projects and an employee of NASA MSFC for over 32 years where he has received numerous awards including the NASA Outstanding Leadership Medal (2017). Formerly he was the Program Manager for the Chandra Observatory (20122015), Deputy Program Manager for Chandra (2010-2012) and the GRAIL Mission Manager (2009-2010). Mr. Baggett has a Bachelor of Science in Electrical Engineering from Auburn University.

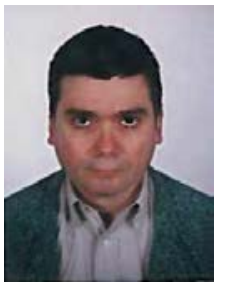

Primo Attina is the I2T Project Manager for IXPE (ASI/INAF) and a member of the IXPE Risk Management Board. Mr. Attina is also the System Manager for the X-ray polarimeter on board $X$-ray Imaging Polarimetry Explorer (XIPE). Formerly $M r$. Attina was the Industrial Program manager of PLATO (TAS-I), the ESA selected Cosmic Vision M3 mission and the Payload manager of BeppoSAX Italian-Dutch mission. He has 31 years of experience in scientific space systems plus 4 years in IT R\&D. Mr. Attina has a Master of Science in Physics from the University of Turin, Italy.

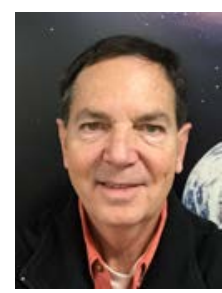

Mike Bowen is the IXPE Configuration Management Lead and serves as Secretariat for the IXPE Risk Management Board. He has worked as a contractor for MSFC NASA for 30 years in Configuration \& Data Management (CDM) and Systems Engineering (SE). $M r$. Bowen has served as the CDM and SE Lead on the Space Shuttle/Redesigned Solid Rocket Motor, provided CDM and SE support to Space Launch System and served as the CDM Lead for the Lightning Imaging Sensor and the Life Sciences Glovebox. He holds a Bachelors in Business Administration and Marketing from Auburn University.

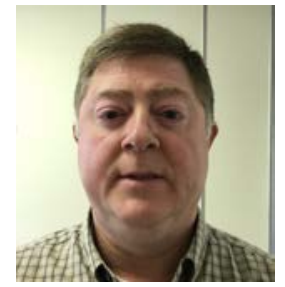

Chris Cowart is the IXPE Chief Safety Officer and a member of the IXPE Risk Management Board. He has worked for over 25 years at MSFC NASA in Safety Engineering. Formerly he was the Chief Safety Officer for SLS Spacecraft/Payload Integration and Evolution Office, Panel Chair for MSFC Space Shuttle Safety and Engineering Review Panel, and Safety Engineer for the Environmental Control and Life Support System (ECLSS). Mr. Cowart has a Bachelor of Science in Electrical Engineering from the University of Alabama in Birmingham.

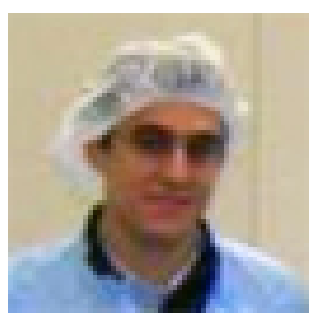

Ettore Del Monte is the I2T Lead Systems Engineer for IXPE and member of the IXPE Risk Management Board. Dr. Del Monte received his Ph.D. in Astronomy in 2005 at the University of Roma "Tor Vergata" (Italy). He worked in the development, qualification, integration and calibration of the SuperAGILE $X$-ray instrument on the AGILE satellite mission, launched in 2007. After AGILE, he worked in proposals for satellite-borne $X$-ray instrumentation like the Large Observatory For x-ray Timing (LOFT) and the X-ray Imaging Polarimetry Explorer (XIPE). Between 2015 and 2017 he was the coordinator of the grant COMpton Polarimeter with Avalanche Silicon readout (COMPASS), given by the Istituto Nazionale di AstroFisica (INAF) in Italy. Since 2009 he is staff member of the Istituto di Astrofisica e Planetologia Spaziali (IAPS) of the Istituto Nazionale di AstroFisica (INAF) in Rome (Italy).

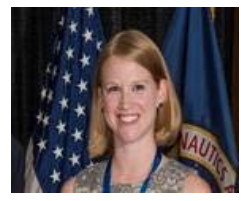

Lindsey Ingram is the Business Manager for IXPE and an ad hoc member of the IXPE Risk Board. Prior to joining IXPE Ms. Ingram, supported the director of the Resource Management Office for Human Exploration \& Operations Mission Directorate at NASA Headquarters. She served as integration lead for the Technical Capability Assessment/Technical Capability Leadership teams on behalf of the Marshall Space Flight Center. Ms. Ingram is the recipient of the NASA Exceptional Achievement Medal and the NASA Space Flight Awareness Trailblazer Award. Ms. Ingram holds a B.S. in Industrial and Systems Engineering and an M.S.E degree in Engineering Management, both from the University of Alabama in Huntsville.

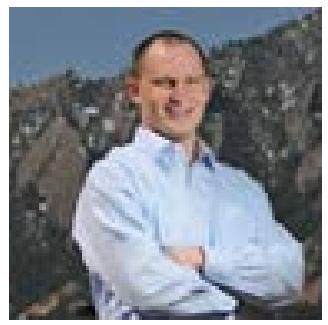

Bill Kalinowski is a Principal Engineer in Electrical Systems Engineering for Ball Aerospace. Mr. Kalinowski currently is the Ball Spacecraft Systems Engineer, spacecraft CAM, C\&DH lead on the NASA IXPE Project and serves as a Risk Owner for spacecraft related risks for IXPE Risk Managment. He was the C\&DH Lead on the Green Propellant Infusion Mission. Before coming to Ball Aerospace, he worked on the Orion MPCV avionics and thermal protection systems as a test and systems engineer with Stellar Solutions. Mr. Kalinowski has performed various other systems engineering and electrical design roles on the Deep Underground Science and Engineering Laboratory (DUSEL), multiple biological experiments flown aboard ISS and STS, general aviation products, and multiple commercial and government satellite systems. Mr. Kalinowski holds B.S. and M.S. degrees in Aerospace Engineering, as well as an M.E. degree in Engineering Management, all from the University of Colorado at Boulder. 


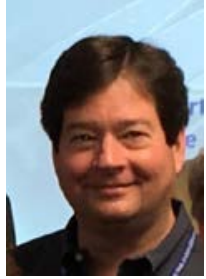

Anthony Kelley is the Project Systems Engineer for IXPE, and is a member of the IXPE Risk Management Board serving as the alternate Chair. Mr. Kelley has over 32-years of experience working space flight systems and payloads, and has received federal, state, and NASA center awards including MSFC Invention of the Year in 2007, 2015 and 2017 and Federal Laboratory Consortium Excellence in Technology Transfer in 2010. Some of Mr. Kelley's previous work includes: Launch Vehicle Lead System Engineer (LSE) for Commercial Crew Program, Lead Engineer for DesignBuild-Test payloads on 5 sounding rockets, Integration Lead for SLS Engine Section Structural Test, Team Lead for Advanced Capabilities \& R\&D in cryogenic fluid management, and COTR for DC-XA Add-on Avionics. Mr. Kelley holds a Bachelor of Science in Electrical Engineering from the University of Alabama in Huntsville (1989) and a Masters of Engineering in Operations Research and Industrial Engineering from Cornell University (1994).

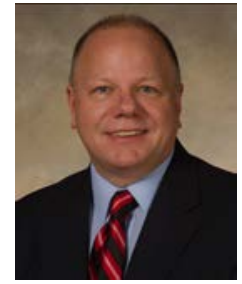

Steven Pavelitz is the Payload Manager for IXPE and a member of the IXPE Risk Management Board. He is the former Project Manager of the International Space Station (ISS) Lightning Imaging Sensor (LIS). Mr. Pavelitz has over 15 years of experience in system engineering and project management of space flight projects at NASA MSFC both as a civil servant and a Sverdrup Technology employee. He has received numerous Outstanding Performance Awards, NASA Group Achievement Awards, and a NASA Silver Snoopy Award. Mr. Pavelitz has a B.S. in Aerospace Engineering from Pennsylvania State University.

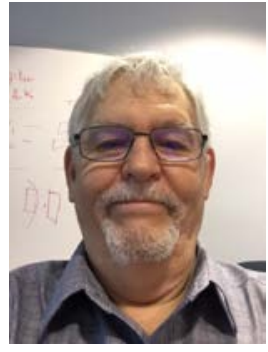

Martin C. Weisskopf is the Principle Investigator for IXPE and a member of the IXPE Risk Management Board. Dr. Weisskopf is Project Scientist for NASA's Chandra X-ray Observatory, and senior scientist for $X$-ray Astronomy in the Space Science Office at the Marshall Center. Known internationally, Dr. Weisskopf has dedicated four decades of his career to ensuring the scientific integrity of NASA's Chandra program. Launched in 1999, the telescope - one of NASA's four "Great Observatories" - continues to deliver unprecedented images of exploding stars, distant galaxies and virtually all other categories of astronomical object.

During his NASA career, Dr. Weisskopf has held a wide array of special appointments and served on numerous committees dedicated to advancing breakthroughs in $X$ ray astronomy. Dr. Weisskopf joined NASA as a senior Xray astronomer in 1977 after nine years teaching at Columbia University in New York City, where he performed many pioneering experiments in X-ray astronomy. He is a fellow of the American Physical Society and of the International Society for Optical Engineering (SPIE).

Dr. Weisskopf earned a bachelor's degree in physics in 1964 from Oberlin College in Oberlin, Ohio, and a doctorate in physics in 1969 from Brandeis University in Waltham, Mass. The author or co-author of over 300 publications, Dr. Weisskopf has received numerous awards and accolades from NASA, academia and professional societies including the Rossi Prize. 


\section{APPENDiX A \\ IXPE RISK REGISTER INPUTS}

An Excel-based risk management tool is used to document and track Project risks and risk mitigation actions, as well as issues and opportunities. The picture below is a snapshot of the IXPE risk register for active risks (i.e. still being tracked, not closed) which shows the input categories.

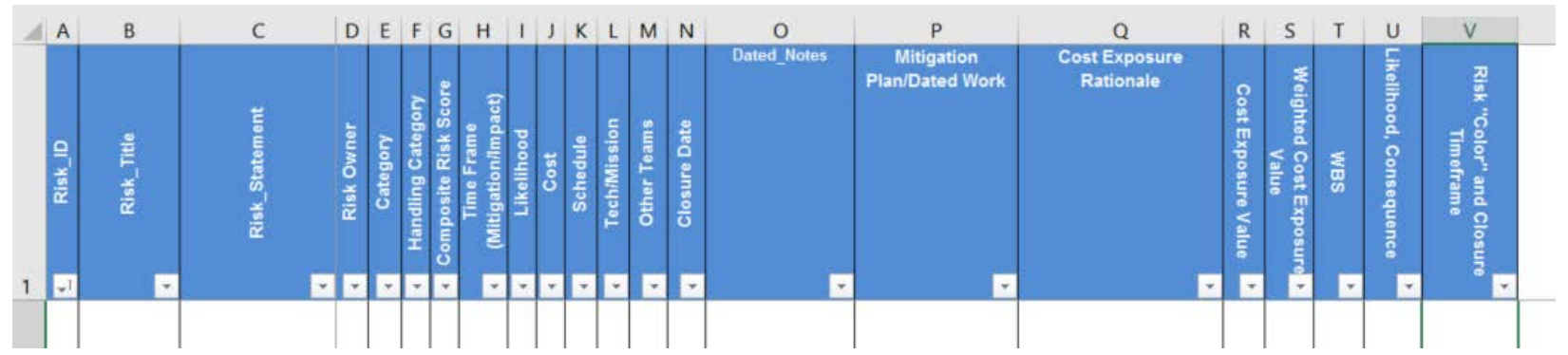

\section{APPENDIX B \\ IXPE DRAFT RISK INPUTS}

The picture below is a snapshot of a draft risk input form and shows the inputs needed to bring a draft risk before the RMB.

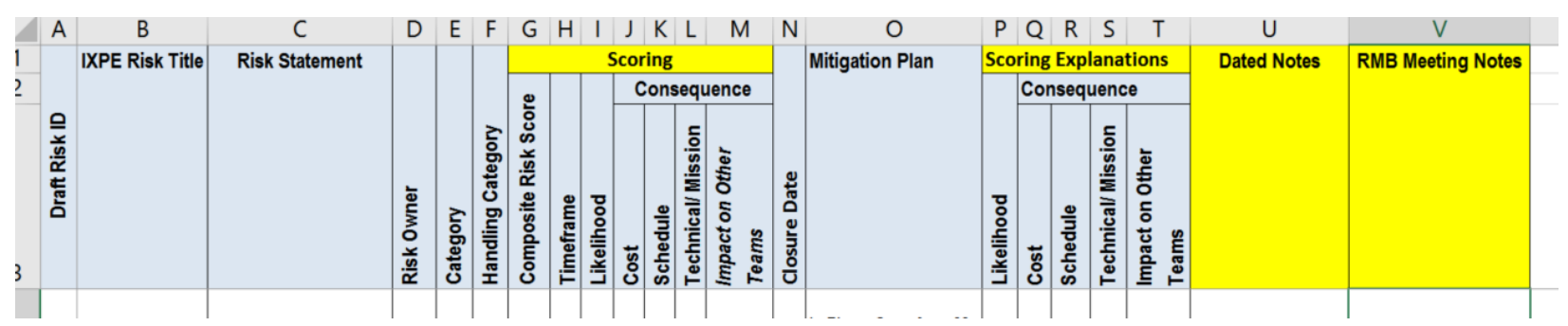

